\title{
Optimal fees in internet auctions
}

\author{
Alexander Matros • Andriy Zapechelnyuk
}

Received: 7 September 2007 / Accepted: 18 April 2008 / Published online: 16 May 2008

(C) Springer-Verlag 2008

\begin{abstract}
An auction house runs a second-price auction with a possibility of resale through re-auctions. It collects listing and closing fees from the seller. We find the fees which maximize the revenue of the auction house. In particular, we show that the optimal listing fee is zero. Our findings are consistent with the policies of eBay, Amazon, Yahoo, and other Internet auctions.
\end{abstract}

Keywords Internet auctions - Auctions with resale $\cdot$ Auction house $\cdot$ Listing fee Closing fee

JEL Classification $\mathrm{D} 44 \cdot \mathrm{C} 78 \cdot \mathrm{D} 82$

\section{Introduction}

An auction is typically viewed as a trade mechanism which involves two sides: sellers and buyers. However, the presence of a third side, an independent mediator, may have essential influence on the strategic behavior of the traders. This is the case in Internet auctions where the role of mediators is played by such giant commercial institutions as eBay, Yahoo, Amazon, and others. In contrast to the existing literature on "optimal auctions," that is, auction mechanisms which maximize a seller's revenue, we are looking for the optimal strategy for a mediator (an "auction house"). This question

\footnotetext{
A. Matros $(\varangle)$

Department of Economics, University of Pittsburgh, Pittsburgh, PA, USA

e-mail: alm75+@pitt.edu
}

A. Zapechelnyuk

Kyiv School of Economics and Kyiv Economics Institute, Kyiv, Ukraine 
has not only theoretic interest, but also practical relevance, as, for example, the leader of the Internet auction industry, eBay Inc., reported net revenue of \$6 billion in 2006. ${ }^{1}$

Usually an auction house requires a seller to pay a listing fee, a price of initiating the auction, and, if the object is sold, a closing fee, a certain fraction of the final price. Our central question is: What are the fees that maximize the profit of the auction house? Generally, in a two-part tariff scheme both tariffs are positive in equilibrium. In this paper, however, we show that the optimal listing fee is zero.

We consider a Vickrey auction with reserve price, an analogue to the proxi-bidding mechanism used by Internet auctions. We assume that if the seller's reserve price is not met, he has a possibility to re-auction the object. This option is available to agents in real life and has essential impact on their strategic behavior (see, e.g., Milgrom 1987; Haile 2000, 2003). In every (re)auction the seller faces a different set of bidders drawn from a large population. The auction house requires the seller to pay a listing fee (which can be negative), and, if the object is sold, a closing fee (a percentage of the closing price). The fees are selected by the auction house before trade and remain constant thereafter.

We characterize the optimal strategy (the choice of the fees) for the auction house. In particular, we show that the optimal listing fee is zero, that is, for the auction house it is not optimal to demand an up-front payment from the seller, nor to offer him a subsidy. Our results provide formal grounds for the current policy of large Internet auction houses. For example, eBay charges the listing fee of $\$ 0.20$ to $\$ 4.80$ (for reserve price above $\$ 500)^{2}$; Amazon charges the listing fee of $\$ 0.10$, which is waived for qualified sellers $^{3}$; QXL.com charges the listing fee of $£ 0.05-0.40$ (for reserve price above $£ 30$ ); Bidville.com and uBid.com charge no listing fees.

This paper is one of the first in the auction literature which raises the question of profit maximization for an auction mediator. Myerson and Satterthwaite (1983) are the first who analyze a bilateral trade mediated by a "broker" in a static environment without possibility of resale. They describe a mechanism which maximizes the broker's payoff subject to individual rationality and incentive compatibility constraints for the traders. A variety of work extends Myerson and Satterthwaite (1983) to the study of two-sided markets (a mass interaction of multiple sellers and buyers, typically with network externalities) mediated by "platforms", starting with the double auction of Wilson (1985) and including (but not limited to) Rochet and Tirole (2003), Hagiu (2004), Reisinger (2004), and Armstrong (2006). Instead, the focus of this paper is the mediated auction-kind interaction of one seller and many buyers.

A large set of related literature deals with unmeditated auctions with the possibility of after-auction resale. Such a possibility affects nontrivially the bidding behavior, since bidders' valuations partially depend on expectations of a resale outcome. There are two approaches: one-time after-auction resale, where the object owner can sell the object after the auction on a secondary market (Milgrom 1987; Bikhchandani and Huang 1989; Gupta and Lebrun 1999; Haile 1999, 2000, 2003;

\footnotetext{
1 See http://investor.ebay.com/releases.cfm.

2 Basic fees can be found on http://pages.ebay.com/help/sell/fees.html.

3 Amazon's fees can be found on http://www.amazon.com/gp/help/customer/display.html.
} 
Krishna 2002, Sect. 4.4; Calzolari and Pavan 2003; Garratt and Tröger 2006), and re-auctioning, where the object may be re-auctioned multiple times (McAfee and Vincent 1997; Horstmann and LaCasse 1997; Zheng 2002).

In this paper we allow re-auctioning, but the important difference from the literature above is that the seller deals with a new set of bidders in every (re)auction. ${ }^{4}$ It entails that the seller faces ex-ante the same problem in every auction, hence, if he is willing to auction the object once, he will re-auction it until the object is sold. This provides some intuition why zero listing fee is optimal: By demanding only a closing fee, the auction house takes the same part in the (expected) current-period payoff and in the continuation payoff of the seller, thus making the interests of the seller and the auction house fully aligned.

The paper is organized as follows. The model is described in Sect. 2. The first step of the analysis, the optimal behavior of the seller, is made in Sect. 3. Our main results concerning the optimal policy of the auction house are stated in Sect. 4. The model assumptions and possible variations are discussed in Sect. 5.

\section{The model}

Consider a seller who has a single object for sale, a large population of bidders, $N$, and an auction house (auction mediator). All bidders in population $N$ have private values for the object, i.i.d. random variables with a distribution function $F$ on interval $[0,1]$. The seller also has a private value independent of bidders' values, with distribution $F_{S}$ on $[0,1]$. Every agent knows her own private value. We assume that functions $F$ and $F_{S}$ are differentiable and have positive density on $(0,1)$. In addition, we assume that $F$ and $F_{S}$ satisfy Myerson's (1981) regularity conditions, namely, $z-\frac{1-F(z)}{f(z)}$ and $z+\frac{F_{s}(z)}{f_{s}(z)}$ are strictly increasing on $(0,1)$, where $f$ and $f_{s}$ denote the corresponding density functions.

The auction house, the seller, and the bidders are engaged in the following game. In period 0 , the auction house announces fees for all subsequent auctions: a listing fee, $c \in \mathbb{R}$, and a closing fee, a fraction $\mu \in[0,1]$ of the closing price (if the object is sold). A negative listing fee means that the auction house pays to the seller amount $|c|$ each time the object is displayed on the auction. In every period $t=1,2, \ldots$ the following two-stage game is played:

Stage 1. The seller decides whether to consume the object or to offer it for sale via auction. If the object is consumed, the game ends. Otherwise, the second stage comes. Stage 2. The auction house runs a Vickrey auction. The set of $k$ bidders is randomly drawn from the population $N$. The seller announces a reserve price, then the bidders make their bids simultaneously. If there is a winner in the auction, she transfers to the seller a payment equal to the second highest bid (or the reserve price) and the game ends. Otherwise, if all bids are below the reserve price, the game proceeds to the next

\footnotetext{
4 An exception is Haile (1999) who assumes that new buyers arrive to a resale market. However, he considers the model of one-time resale (not re-auction), and, besides, he assumes that the bidders participating in the first period remain in the secondary market.
} 
period. Regardless of the auction outcome, the seller pays to the auction house the listing fee $c$, and in addition, if the object is sold, the closing fee $\mu$.

We assume that all agents are risk neutral, the seller does not derive any utility from the object until it is sold or consumed, $\delta$ is a discount factor for all agents and the auction house, parameters $k, \delta$, and the distribution functions $F$ and $F_{S}$ are common knowledge.

In the next section we find the optimal seller's reserve price, $r_{c, \mu}^{*}$, for any auction house fee scheme $(c, \mu)$. Then, in Sect. 4 we find the optimal auction house fee scheme.

\section{Seller: optimal reserve price}

Fix the auction fee scheme $(c, \mu)$ and period $t$. To simplify notations, we omit the time period index whenever it does not result in a confusion.

For every reserve price $r \in[0,1]$ denote by $q(r)$ the probability that the object is sold on the auction, and by $P(r)$ the expected closing price, i.e., the expected payment of the winning bidder conditional on the event that the object is sold.

Denote by $v\left(r, v_{c, \mu}^{*}\right)$ the expected seller's revenue from the auction, where $r \in[0,1]$ is the selected reserve price and $v_{c, \mu}^{*} \in \mathbb{R}$ is the highest expected revenue that the seller can attain from the next-period auction. Thus,

$$
v\left(r, v_{c, \mu}^{*}\right) \equiv q(r) P(r)(1-\mu)+(1-q(r)) \delta v_{c, \mu}^{*}-c .
$$

Since in every period the seller faces ex-ante the same bidding environment, we consider only Markov strategies of the seller, i.e., strategies which depend on the seller's private value of the object and are independent of the decision time. The following equation must hold,

$$
v_{c, \mu}^{*}=\max _{0 \leq r \leq 1} v\left(r, v_{c, \mu}^{*}\right)
$$

We can now describe the optimal (behavior) strategy of the seller. Let $v_{s}$ be the seller's private value of the object. Then

(a) The seller consumes the object immediately (in period 1) if his private value is at least as high as the expected gain from the auction (net of the auction fees)

$$
v_{s} \geq v_{c, \mu}^{*}
$$

otherwise he auctions the object in every subsequent period, until it is sold. In particular, it means that if $\mu=1$, then $v_{c, \mu}^{*}<0 \leq v_{s}$, or the seller consumers the object.

(b) If the seller auctions the object, $\mu<1$, then in every period the reserve price is

$$
r_{c, \mu}^{*} \in \underset{0 \leq r \leq 1}{\operatorname{argmax}} v\left(r, v_{c, \mu}^{*}\right)=\underset{0 \leq r \leq 1}{\operatorname{argmax}}\left[q(r)\left(P(r)-\frac{\delta v_{c, \mu}^{*}}{1-\mu}\right)\right] .
$$


Note that the seller's optimal reserve price $r_{c, \mu}^{*}$ and expected revenue $v_{c, \mu}^{*}$ do not depend on his private value $v_{s}$ (as long as $v_{s}<v_{c, \mu}^{*}$ ).

The following lemma helps to prove the main result of this section.

Lemma 1 For every $z \in \mathbb{R}$ function $q(r)(P(r)-z)$ is maximized w.r.t. $r$ on $[0,1]$ at a unique point.

Proof Consider a Vickrey auction with $k$ bidders. By a straightforward computation (see, e.g., Krishna 2002, Sects. 2.2 and 2.4), we obtain

$$
\begin{aligned}
q(r) & =1-F^{k}(r), \text { and } \\
P(r) & =\frac{1}{1-F^{k}(r)}\left[\left(G(r)-F^{k}(r)\right) r+\int_{r}^{1} x d G(x)\right],
\end{aligned}
$$

where $G$ is the distribution function of the second highest bidder's value

$$
G(x)=F^{k}(x)+k F^{k-1}(x)(1-F(x)) .
$$

Then, for any constant $z$, integrating $\int_{r}^{1} x d G(x)$ by parts yields

$$
q(r)(P(r)-z)=1-z+F^{k}(r)(z-r)-\int_{r}^{1} G(x) d x .
$$

Taking the derivative w.r.t. $r$, we obtain

$$
\frac{\partial}{\partial r}[q(r)(P(r)-z)]=-k F^{k-1}(r) f(r)\left[r-\frac{1-F(r)}{f(r)}-z\right] .
$$

Let $r^{*} \in \operatorname{argmax}_{r \in[0,1]} q(r)(P(r)-z)$. Since $k F^{k-1}(r) f(r)>0$ for all $r \in(0,1]$ and by Myerson's regularity condition $r-\frac{1-F(r)}{f(r)}$ strictly increases, it follows that $r^{*}=0$ if $r-\frac{1-F(r)}{f(r)}>z$ for all $r \in(0,1], r^{*}=1$ if $r-\frac{1-F(r)}{f(r)}<z$ for all $r \in[0,1)$, otherwise $r^{*}$ is the unique value which satisfies $r^{*}-\frac{1-F\left(r^{*}\right)}{f\left(r^{*}\right)}=z$.

We can now state the main result of this section.

Proposition 1 For every pair of auction fees $(c, \mu) \in \mathbb{R} \times[0,1]$ there exists a unique $\operatorname{pair}\left(r_{c, \mu}^{*}, v_{c, \mu}^{*}\right) \in[0,1] \times \mathbb{R}$ which satisfies

$$
v_{c, \mu}^{*}=v\left(r_{c, \mu}^{*}, v_{c, \mu}^{*}\right)=\max _{0 \leq r \leq 1} v\left(r, v_{c, \mu}^{*}\right) .
$$

Proof If the seller auctions the object, $\mu<1$, then (1) can be rewritten as follows

$$
v\left(r, v_{c, \mu}^{*}\right)=\delta v_{c, \mu}^{*}-c+(1-\mu)\left[q(r)\left(P(r)-\frac{\delta v_{c, \mu}^{*}}{1-\mu}\right)\right] .
$$


After maximizing (5) w.r.t. $r$ on $[0,1]$, moving $\delta v^{*}$ to the left-hand side and dividing both sides by $(1-\delta)$, we obtain

$$
v_{c, \mu}^{*}=-\frac{c}{1-\delta}+\frac{1-\mu}{1-\delta} \max _{0 \leq r \leq 1}\left[q(r)\left(P(r)-\frac{\delta v_{c, \mu}^{*}}{1-\mu}\right)\right] .
$$

Note that if $v_{c, \mu}^{*}=-\frac{c}{1-\delta}$, then the right-hand side is greater than the left-hand side; if $v_{c, \mu}^{*}=\frac{1-\mu}{\delta}$, then the right-hand side is less than the left-hand side. Since the lefthand side strictly increases and the right-hand side weakly decreases in $v_{c, \mu}^{*} \in \mathbb{R}$, there exists a unique solution of the Eq. (6).

Applying Lemma 1 with $z=\frac{\delta v_{c, \mu}^{*}}{1-\mu}$ yields a unique value of $r_{c, \mu}^{*}$.

This proposition shows that the seller auctions the object and sets the reserve price $r^{*}$ regardless of his use value of $v_{s}$, as long as $v_{s}<v_{c, \mu}^{*}$. Thus, the equilibrium strategy of the seller is pooling: it is not strictly monotonic with respect to his use value. The effective reserve value of the seller is not his private use value, instead, it is the continuation value, the expected payoff from the future auctions.

Note that the number of bidders drawn in every period does not have to be deterministic; it suffices to have a random number of bidders drawn from the same distribution in every period. Indeed, since the seller selects a reserve price before he knows how many bidders participate in the auction, his decision depends only on the distribution of the number of bidders.

\section{Auction house: optimal fees}

Fix $\left(r_{c, \mu}^{*}, v_{c, \mu}^{*}\right)$, and suppose that $v_{s}<v_{c, \mu}^{*}$. Denote by $U\left(r_{c, \mu}^{*}\right)$ the expected gross revenue of the seller (before the fees are deducted). Since $r_{c, \mu}^{*}$ is constant over time, we can write $U\left(r_{c, \mu}^{*}\right)$ as follows,

$$
U\left(r_{c, \mu}^{*}\right)=q\left(r_{c, \mu}^{*}\right) P\left(r_{c, \mu}^{*}\right)+\left(1-q\left(r_{c, \mu}^{*}\right)\right) \delta U\left(r_{c, \mu}^{*}\right) .
$$

The (unconditional) expected revenue of the auction house can be written as the product of the expected revenue of the auction house conditional on $v_{s}<v_{c, \mu}^{*}$ and the probability that $v_{s}<v_{c, \mu}^{*}$,

$$
\left(U\left(r_{c, \mu}^{*}\right)-v_{c, \mu}^{*}\right) F_{S}\left(v_{c, \mu}^{*}\right) .
$$

First, we find a pair $\left(r_{c, \mu}^{*}, v_{c, \mu}^{*}\right)$ which maximizes (8). Then we derive the optimal scheme $(c, \mu)$ which yields the desired $\left(r_{c, \mu}^{*}, v_{c, \mu}^{*}\right)$.

Denote by $\bar{U}$ the highest expected gross revenue of the seller,

$$
\bar{U}=\max _{0 \leq r \leq 1}[q(r) P(r)+(1-q(r)) \delta \bar{U}] .
$$

Note that $\bar{U}$ is independent of $(c, \mu)$.

We can now state the main result of the paper. 
Theorem 1 Under the optimal fee scheme, the expected revenue of the auction house is equal to

$$
\max _{v^{*} \in[0,1]}\left(\bar{U}-v^{*}\right) F_{S}\left(v^{*}\right) .
$$

The unique pair of optimal fees $(c, \mu)$ is given by $c=0$ and $\mu=\frac{\bar{U}-v^{*}}{\bar{U}}$, where $v^{*}$ is the maximizer of (10).

Proof Let $\left(r^{*}, v^{*}\right)$ be a pair which maximizes (8). First, we show that such a pair is unique. Notice that the only part of (8) which depends on $r^{*}$ is $U\left(r^{*}\right)$, hence, $r^{*} \in \operatorname{argmax}_{0 \leq r \leq 1} U(r)$ subject to (7) with replacement of $r_{c, \mu}^{*}$ by $r^{*}$. By (9) this immediately yields $U\left(r^{*}\right)=\bar{U}$, and

$$
v^{*} \in \underset{v \in[0,1]}{\operatorname{argmax}}(\bar{U}-v) F_{S}(v)
$$

Taking derivative of $W(v) \equiv(\bar{U}-v) F_{s}(v)$, we obtain

$$
\frac{d W(v)}{d v}=f_{s}(v)\left(\bar{U}-\left(v+\frac{F_{s}(v)}{f_{s}(v)}\right)\right) .
$$

Since $f_{s}(v)>0$ and, by Myerson's regularity condition, $v+\frac{F_{s}(v)}{f_{s}(v)}$ is strictly increasing, there exists a unique maximizer of $W(v), v^{*}$. Next, rewriting (9) as

$$
(1-\delta) \bar{U}=\max _{0 \leq r \leq 1} q(r)(P(r)-\delta \bar{U})
$$

and applying Lemma 1 with $z=\delta \bar{U}$, we obtain

$$
r^{*}=\underset{0 \leq r \leq 1}{\operatorname{argmax}}[q(r)(P(r)-\delta \bar{U})] .
$$

We now derive the optimal scheme $(c, \mu)$ which yields $\left(r_{c, \mu}^{*}, v_{c, \mu}^{*}\right)=\left(r^{*}, v^{*}\right)$. The seller chooses the reserve price $r_{c, \mu}^{*}$ which maximizes his revenue,

$$
r_{c, \mu}^{*}=\underset{0 \leq r \leq 1}{\operatorname{argmax}}\left[q(r)\left(P(r)-\frac{\delta v^{*}}{1-\mu}\right)\right] .
$$

Combining (11) and (12) yields that $r_{c, \mu}^{*}=r^{*}$ if and only if

$$
\mu=\frac{\bar{U}-v^{*}}{\bar{U}} .
$$

Multiplying both sides of (9) by $1-\mu$, we obtain

$$
(1-\mu) \bar{U}=\max _{0 \leq r \leq 1}[q(r) P(r)(1-\mu)+(1-q(r)) \delta \bar{U}(1-\mu)] .
$$


Since (13) can be written as $v^{*}=(1-\mu) \bar{U}$, we have

$$
v^{*}=\max _{0 \leq r \leq 1}\left[q(r) P(r)(1-\mu)+(1-q(r)) \delta v^{*}\right],
$$

but by (1)

$$
v_{c, \mu}^{*}=\max _{0 \leq r \leq 1}\left[q(r) P(r)(1-\mu)+(1-q(r)) \delta v_{c, \mu}^{*}\right]-c .
$$

Hence, $v_{c, \mu}^{*}=v^{*}$ if and only if $c=0$.

Theorem 1 states that the optimal fee scheme for the auction house is a positive percentage of the seller's revenue and zero listing fee. The percentage is selected in such a way that makes interests of the seller and the auction house fully aligned. By demanding a percentage of the seller's payoff, the auction house takes the same part in the (expected) current-period payoff and in the continuation payoff. It aligns the seller's and the auction house's interests not only in the current period, but also in the future.

Note that in the optimal fee scheme, zero listing fee means that the seller neither collects an up-front payment from the seller, nor subsidizes the seller.

\section{Discussion}

We discuss our basic assumptions in this section.

\section{No re-auction possibility for a winning bidder.}

In our model a winning bidder is not allowed to re-auction the object. Adding this possibility for a winning bidder would not make any effect on the optimal reserve price for the (initial) seller and on the optimal auction fees.

\section{No outside trade.}

It is crucial for our analysis that all trade happens via the auction. Namely, the seller does not use the auction as a free advertising, for instance, by setting the reserve price high enough to make sure that no one buys the object. The typical case when this assumption is violated is the Internet auctions for used cars.

\section{Auction house monopoly.}

We assume that the auction house is a monopolist. This assumption can be considered as an approximation of reality, since large auction houses tend to gain substantial monopolistic power. For example, a seller's rating based on positive feedback over completed sales motivates the seller to stay with the same auction house. The size of an auction house also contributes to its monopolistic power: bigger auction houses are more attractive to traders.

\section{Listing fee and closing fee.}

The use of listing and closing fees is the most popular way for the auction houses to obtain payments. In fact, we are not aware of any other payment mechanism which is used in Internet auctions. Our analysis demonstrates that the actual listing and closing fees that major Internet auction houses apply indeed maximize their revenue. 
Acknowledgements We are grateful to the Editor-in-Chief, Atila Abdulkadiroglu, an anonymous referee, and an associate editor for their comments.

\section{References}

Armstrong M (2006) Competition in two-sided markets. RAND J Econ 37:668-691

Bikhchandani S, Huang C (1989) Auctions with resale markets: an exploratory model of treasury bill markets. Rev Financial Stud 2:311-339

Calzolari G, Pavan A (2003) Monopoly with resale. Working paper 2003.20, Fondazione Eni Enrico Mattei

Garratt R, Tröger T (2006) Speculation in standard auctions with resale. Econometrica 74:753-769

Gupta M, Lebrun B (1999) First price auctions with resale. Econ Lett 64:181-185

Hagiu A (2004) Optimal pricing and commitment in two-sided markets. Working paper, Department of Economics, Princeton

Haile PA (1999) Auctions with resale. Working paper 33, Social Sciences, Wisconsin Madison

Haile PA (2000) Partial pooling at the reserve price in auctions with resale opportunities. Games Econ Behav 33:231-248

Haile PA (2003) Auctions with private uncertainty and resale opportunities. J Econ Theory 108:72-110

Horstmann IJ, LaCasse C (1997) Secret reserve prices in a bidding model with a resale option. Am Econ Rev 87:663-684

Krishna V (2002) Auction theory. Academic Press, New York

McAfee RP, Vincent D (1997) Sequentially optimal auctions. Games Econ Behav 18:246-276

Milgrom P (1987) Auction theory. In: Bewley T (ed) Advances in economic theory: fifth world congress. Cambridge University Press, Cambridge, pp 1-32

Myerson R (1981) Optimal auction design. Math Oper Res 6:58-73

Myerson R, Satterthwaite M (1983) Efficient mechanisms for bilateral trading. J Econ Theory 29:265-281

Reisinger M (2004) Two-Sided Markets with Negative Externalities. Department of Economics, University of Munich, discussion paper 2004-27

Rochet J, Tirole J (2003) Platform competition in two-sided markets. J Euro Econ Assoc 1:990-1029

Wilson R (1985) Incentive efficiency of double auctions. Econometrica 53:1101-1115

Zheng C (2002) Optimal auction with resale. Econometrica 70:2197-2224 